Pesq. Vet. Bras. 37(10):1153-1158, outubro 2017 DOI: $10.1590 / \mathrm{S} 0100-736 \mathrm{X} 2017001000018$

\title{
Incidência de Candida spp. segundo a sazonalidade em excretas de aves silvestres e exóticas cativas analisadas no Laboratório ADN/Poços de Caldas-MG, Brasil (2010-2014) ${ }^{1}$
}

\author{
Jackeline N. Alves ${ }^{2,3 *}$, Lilian B. Elston ${ }^{2}$ e Marcelo L. Brisola ${ }^{2}$
}

\begin{abstract}
Alves J.N., Elston L.B. \& Brisola M.L. 2017. [Seasonal incidence of Candida spp. found in feces of captive wild and exotic birds by ADN Laboratory/Poços de Caldas-MG, Brazil (2010-2014).] Incidência de Candida spp. segundo a sazonalidade em excretas de aves silvestres cativas analisadas no Laboratório ADN/Poços de Caldas-MG, Brasil (2010-2014). Pesquisa Veterinária Brasileira 37(10):1153-1158. Faculdade de Medicina Veterinária, Pontifícia Universidade Católica de Minas Gerais, Campus Poços de Caldas, Av. Padre Francis Cletus Cox 1661, Poços de Caldas, MG 37701-355, Brazil. E-mail: jackeline.an.medvet@gmail.com

Candidiasis is a frequent disease caused by yeasts of Candida spp., that acts either like primary or secondary agent for humans and aviary important disease. This article carried out data analysis from 599 laboratory avian samples sent for microbiology analysis in a commercial diagnostic laboratory located in Poços de Caldas city-Minas Gerais state in Brazil, from 2010 to 2014 period with respect to seasonality and geographic distribution. All avian orders analysis from all geographic areas studied reveled $28.05 \%$ positives results $(168 / 599)$ e $71.95 \%$ negatives results (431/599), distributed by seasonality $19.9 \%$ $(119 / 599)$ at summer, $30.6 \%(183 / 599)$ in autumn , 28.04\% (168/599) in winter and 21.54\% (129/599) in springs. At summer 31.09\% (37/119) were positives for Candida sp.; $31.15 \%(57 / 183)$ in autumn; 30.4\% (51/168) in winter at last $17.83 \%(23 / 129)$ in springs. Results revealed at Q-square statistic test $(p \leq 0.05)$ significant reduction in occurrence at springs ( $p=0.03$ ) possible due to an increased immunocompetence at this time but more studies are necessary to better understanding this finding.
\end{abstract}

INDEX TERMS: Candida spp., candidiasis, mycosis, zoonosis, microbiological diagnosis, brazilian wild birds.

RESUMO.- Candidíase é a doença causada pelas leveduras do gênero Candida spp., agindo tanto como agentes primários ou secundários de doenças importantes em aves e humanos. 0 presente estudo teve por objetivo estudar as 599 amostras com pedido de diagnóstico para Candida spp. em um laboratório diagnóstico comercial na cidade de Poços de Caldas, MG-Brasil, no período de 2010 à 2014, levando em consideração a sazonalidade (verão, outono, inverno e primavera) e origem geográfica das amostras.

\footnotetext{
${ }^{1}$ Recebido em 23 de julho de 2015.

Aceito para publicação em 9 de dezembro de 2016.

${ }^{2}$ Faculdade de Medicina Veterinária, Pontifícia Universidade Católica de Minas Gerais, Campus Poços de Caldas (PUC-Minas), Av. Padre Francis Cletus Cox 1661, Poços de Caldas, MG 37701-355, Brasil. *Autor para correspondência: jackeline.an.medvet@gmail.com

${ }^{3}$ Aluna de Graduação do Curso de Medicina Veterinária, PUC-Minas Poços de Caldas, Av. Padre Francis Cletus Cox 1661, Poços de Caldas, MG 37701-355.
}

Ao analisar o grupo com todas as ordens de aves em todo território brasileiro, foram $28,05 \%$ resultados positivos (168/599) e 71,95\% resultados negativos (431/599), sendo 19,9\% (119/599) dos resultados obtidos no verão, $30,6 \%$ (183/599) no outono, 28,04\% (168/599) no inverno e $21,54 \%(129 / 599)$ na primavera. Dentro dos resultados obtidos, no verão $31,09 \%$ (37/119) foram positivos; no outono 31,15\% (57/183); no inverno 30,4\% (51/168) e por fim, na primavera 17,83\% (23/129) (Quadro 3), tendo sido demonstrada baixa incidência nesta última estação $(p=0,003)$ pelo teste de Qui-Quadrado. Com base nestes achados conclui-se que durante a primavera, há diminuição da incidência de resultados positivos para Candida spp. possivelmente devido a um aumento da imunocompetência destes animais durante esta estação, sendo necessários mais estudos para associar resultados clínico-práticos aos estatísticos encontrados nesta pesquisa. 
TERMOS DE INDEXAÇÃO: Candida spp., doenças das aves, candidíase, micose, zoonose, diagnóstico microbiológico, aves silvestres brasileiras.

\section{INTRODUÇÃO}

No Brasil as aves são os animais silvestres mais comercializados devido à preferência destas pelos compradores e por sua vasta variedade de fauna. A maior procura pelo comércio é de psitacídeos, principalmente pelo fato de imitarem a voz humana, pela inteligência e pela beleza. Porém, os passeriformes são as aves mais apreendidas no comércio ilegal devido à facilidade de captura e também pela tradição da criação de pássaros em gaiolas no país, que se estende ao longo de várias décadas (Renctas 2011). 0 Brasil abriga um enorme número de espécies de aves endêmicas, ou seja, somente encontradas neste território, compreendendo mais de 240 espécies neste grupo, portanto a conservação das mesmas depende principalmente dos brasileiros (Machado et al. 2010). Há pouco conhecimento sobre a incidência e distribuição de patógenos causadores de doenças em aves de vida livre e cativas no Brasil, principalmente de fungos infectocontagiosos (Fraga 2014).

Em se tratando de Candida spp., sabe-se que são leveduras cosmopolitas aeróbias obrigatórias, com crescimento em amplas variações de temperatura e $\mathrm{pH}$, sendo destruídas em temperaturas acima de $50^{\circ} \mathrm{C}$, luz ultra violeta, cloro e desinfetantes, como o amônio quaternário, resistentes ao congelamento e sobrevivem muito bem em materiais inanimados e matéria orgânica. Vivem em comensalismo com mamíferos e aves em suas mucosas na maioria das vezes, também no trato genital, pele e unhas (Hirsh \& Zee 2003, Fisher \& Cook 2001, Quinn et al. 2005, Silva 2010).

Candidíase é a doença causada pelas leveduras do gênero Candida spp. (Nouri et al. 2009, Lanteri et al. 2012), agindo como agentes primários ou secundários de doenças importantes em aves e humanos (Coles 2007), ao contrário do que se pensava há alguns anos, em que eram vistas apenas como agentes secundários e oportunistas em indivíduos imuno comprometidos. Quando cultivadas em ágar Sabouraud produzem colônias esbranquiçadas, úmidas e convexas ao serem incubadas de $24-48 \mathrm{~h}$ a $37^{\circ} \mathrm{C}$. (Velasco 2000). Candida albicans, sendo facilmente encontrada na cavidade oral em humanos e também o principal agente causador de aftas nesta espécie (Hazen 1995, Brandão \& Beaufrère 2013), tem sido responsável por várias formas de apresentação de doenças que representam grande risco à sanidade de homens e animais (Hazen 1995). Em aves a candidíase primária de ventrículo é uma doença muito comum, sendo que os sinais clínicos incluem perda de peso, regurgitação e presença de alimentos não digeridos nas fezes (Muir \& Raidal 2012). Além do trato gástrico superior, espécies de Candida spp., também patogênicas ao homem (C. albicans, C. tropicalis, C. famata, C. guilliermondi) podem ser encontradas em excretas de aves de companhia, o que demonstra a importância clínica de serem carreadoras de zoonoses (Cafarchia et al. 2006, Santos et al. 2009).

O notável aumento de criação de aves coloca o Médico Veterinário numa importante posição quanto ao esclarecimento e orientação do proprietário no modo de criação e de alimentação destas espécies. Além destas questões, devido ao maior contato diário com a criação, é de extrema importância a orientação sobre o risco e o controle de zoonoses (Santos et al. 2008).

0 objetivo deste trabalho foi estudar a incidência de resultados microbiológicos positivos para Candida spp. em aves silvestres e exóticas, cujas amostras foram enviadas ao Laboratório ADN/Poços de Caldas-MG, Brasil, no período de 2010 à 2014, levando em consideração a sazonalidade (verão, outono, inverno e primavera), contribuindo para o aumento de dados acerca da frequência de candidíase nestas aves de diferentes espécies e para que o Médico Veterinário possa assumir um maior papel na medicina preventiva da criação destas aves.

\section{MATERIAL E MÉTODOS}

Foram analisados nos arquivos do Laboratório ADN/Poços de Caldas, MG, Brasil todos os laudos de doenças do período de 2010 a 2014, tendo sido encontrados 599 laudos para diagnóstico de Candida spp. de aves silvestres e exóticas, cujas fezes foram enviadas em coletor para exame de fezes a seco (COPRO SECO ${ }^{\circledR}$ Campinas Medical). Foram selecionados para este estudo apenas laudos em que se especificavam a região do Brasil a qual pertenciam, a identificação da espécie das aves e o período em que foram coletadas para análise.

As amostras foram processadas em cultura microbiológica: a semeadura em forma de estrias foi feita próxima a chama de um bico de Bunsen, utilizando alça microbiológica previamente flambada. 0 meio de cultura utilizado foi Ágar Sabouraud Dextrose (NEW PROV ${ }^{\circledR)}$ e, após a semeadura, as amostras foram incubadas em estufa (HEKA-TURBO-BRUTGERÄT ${ }^{\circledR}$ ) por 48 horas a $37^{\circ} \mathrm{C}$. Depois deste período, as placas foram analisadas e diagnosticadas positivas para Candida spp. se houvesse crescimento de colônias esbranquiçadas, úmidas e convexas.

Os 599 laudos para Candida spp. foram divididos em resultados positivos e negativos no verão, outono, inverno e primavera, seguindo as datas do início e término das estações nos anos de 2010 a 2014 de acordo com o Instituto Nacional de Meteorologia (INMET 2015) sendo separados de acordo com a ordem, a família, o gênero e a espécie das aves, seguindo a classificação de Peterson \& Life (1971), Marinho et al. (2010), Piacentini et al. (2015) e Gill \& Donsker (2016) (Quadros 1 e 2).

A diferença entre as épocas do ano foi verificada pelo teste de Qui-Quadrado com Partição, tendo como nível de significância 5\%. Foi utilizado o pacote estatístico BioEstat 5.0 (Ayres et. al. 2007).

Respeitando a ética, a identificação das aves e a identidade de seus proprietários não foram citadas neste estudo.

\section{RESULTADOS}

As 599 amostras com pedido de diagnóstico para Candida spp. foram divididas de acordo com a ordem da aves:

Quadro 1. Datas do início das estações dos anos de 2010 a 2014

\begin{tabular}{lccccc}
\hline & 2010 & 2011 & 2012 & 2013 & 2014 \\
\cline { 2 - 6 } Estação & \multicolumn{5}{c}{ Data/mês } \\
\hline Outono & $20 / 03$ & $20 / 03$ & $20 / 03$ & $20 / 03$ & $20 / 03$ \\
Inverno & $21 / 06$ & $21 / 06$ & $20 / 06$ & $21 / 06$ & $21 / 06$ \\
Primavera & $23 / 09$ & $23 / 09$ & $22 / 09$ & $22 / 09$ & $22 / 09$ \\
Verão & $21 / 12$ & $22 / 12$ & $21 / 12$ & $21 / 12$ & $21 / 12$
\end{tabular}

Adaptado de INMET 2015. 
Quadro 2. Levantamento de resultados positivos e negativos para Candida spp. em aves silvestres e exóticas encontrados entre 2010 e 2014 de acordo com a estação do ano

\begin{tabular}{|c|c|c|c|c|c|c|c|c|}
\hline & \multicolumn{2}{|c|}{ Verão } & \multicolumn{2}{|c|}{ Outono } & \multicolumn{2}{|c|}{ Inverno } & \multicolumn{2}{|c|}{ Primavera } \\
\hline & Positivos & Negativos & Positivos & Negativos & Positivos & Negativos & Positivos & Negativos \\
\hline & \multicolumn{8}{|c|}{ Ordem Psittaciforme } \\
\hline Amazona aestiva & 4 & 1 & 2 & 1 & 1 & 1 & 1 & 3 \\
\hline Amazona amazonica & 0 & 0 & 0 & 0 & 1 & 0 & 0 & 0 \\
\hline Amazona rhodocorytha & 2 & 0 & 0 & 0 & 0 & 0 & 0 & 1 \\
\hline Amazona vinacea & 0 & 2 & 0 & 0 & 1 & 0 & 0 & 0 \\
\hline Anodorhynchus hyacinthinus & 0 & 6 & 0 & 2 & 2 & 0 & 3 & 3 \\
\hline Ara ararauna & 5 & 3 & 4 & 2 & 1 & 5 & 0 & 3 \\
\hline Ara chloropterus & 1 & 2 & 0 & 1 & 1 & 1 & 0 & 0 \\
\hline Ara macao & 1 & 2 & 0 & 1 & 0 & 2 & 0 & 2 \\
\hline Ara rubrogenys & 1 & 1 & 0 & 3 & 0 & 2 & 2 & 2 \\
\hline Aratinga leucophthalmus & 0 & 0 & 0 & 0 & 1 & 1 & 0 & 2 \\
\hline Nymphicus hollandicus & 2 & 7 & 1 & 2 & 2 & 1 & 1 & 6 \\
\hline Platycercus elegans & 0 & 0 & 1 & 0 & 0 & 0 & 0 & 0 \\
\hline Platycercus eximius & 0 & 0 & 1 & 0 & 0 & 0 & 0 & 0 \\
\hline Poicephalus senegalus & 0 & 0 & 1 & 0 & 0 & 0 & 0 & 1 \\
\hline Primolius maracana & 1 & 3 & 1 & 0 & 0 & 0 & 2 & 0 \\
\hline Psittacula krameri & 0 & 0 & 1 & 2 & 0 & 0 & 0 & 6 \\
\hline Psittacus erithacus & 0 & 0 & 0 & 3 & 2 & 1 & 1 & 1 \\
\hline Ramphastos toco & 1 & 1 & 1 & 0 & 3 & 0 & 1 & 2 \\
\hline \multirow[t]{2}{*}{ Trichoglossus haematodus } & 0 & 0 & 3 & 0 & 0 & 0 & 0 & 1 \\
\hline & \multicolumn{8}{|c|}{ Ordem Galliforme } \\
\hline Aburria cujubi & 0 & 0 & 0 & 0 & 1 & 0 & 0 & 0 \\
\hline Chrysolophus pictus & 1 & 0 & 0 & 0 & 1 & 0 & 0 & 0 \\
\hline \multirow[t]{2}{*}{ Crax daubentoni } & 0 & 0 & 0 & 0 & 0 & 0 & 1 & 0 \\
\hline & \multicolumn{8}{|c|}{ Ordem Anseriforme } \\
\hline Anas capensis & 0 & 0 & 0 & 0 & 0 & 0 & 1 & 0 \\
\hline Anhima cornuta & 0 & 0 & 0 & 0 & 0 & 1 & 1 & 0 \\
\hline Branta bernicla & 0 & 0 & 0 & 0 & 1 & 0 & 0 & 0 \\
\hline Chauna torquata & 0 & 0 & 1 & 0 & 0 & 0 & 0 & 0 \\
\hline \multirow[t]{2}{*}{ Tadorna radjah } & 0 & 0 & 0 & 0 & 1 & 0 & 0 & 0 \\
\hline & \multicolumn{8}{|c|}{ Ordem Gruiforme } \\
\hline \multirow[t]{2}{*}{ Balearica regulorum } & 3 & 0 & 2 & 0 & 0 & 0 & 0 & 0 \\
\hline & \multicolumn{8}{|c|}{ Ordem Tinamiforme } \\
\hline Crypturellus obsoletus & 0 & 0 & 0 & 0 & 1 & 1 & 0 & 2 \\
\hline \multirow[t]{2}{*}{ Tinamus solitarius } & 2 & 2 & 0 & 0 & 1 & 1 & 1 & 0 \\
\hline & \multicolumn{8}{|c|}{ Ordem Cuculiforme } \\
\hline Musophaga violacea & 1 & 0 & 0 & 0 & 0 & 0 & 1 & 0 \\
\hline Deroptyus accipitrinus & 0 & 0 & 0 & 1 & 1 & 0 & 0 & 0 \\
\hline Eos bornea & 0 & 0 & 1 & 1 & 0 & 1 & 0 & 0 \\
\hline Guaruba guarouba & 0 & 1 & 0 & 0 & 1 & 0 & 0 & 0 \\
\hline Lorius garrulus & 0 & 0 & 3 & 0 & 0 & 0 & 0 & 1 \\
\hline Lorius lory & 0 & 0 & 2 & 0 & 0 & 0 & 0 & 1 \\
\hline & & & & Ordem Pa & seriforme & & & \\
\hline Saltator similis & 3 & 3 & 4 & 3 & 3 & 4 & 0 & 7 \\
\hline Serinus canaria & 1 & 3 & 5 & 3 & 3 & 7 & 0 & 4 \\
\hline Sporophila angolensis & 4 & 39 & 18 & 90 & 20 & 80 & 4 & 53 \\
\hline Sporophila maximiliani & 0 & 1 & 1 & 10 & 0 & 6 & 2 & 1 \\
\hline Sicalis flaveola & 0 & 2 & 1 & 1 & 0 & 0 & 0 & 0 \\
\hline & & & & rdem Phoer & copterifor & & & \\
\hline Phoenicopterus roseus & 3 & 1 & 2 & 0 & 0 & 0 & 0 & 0 \\
\hline Ordem Musophagiforme & & & & & & & & \\
\hline Tauraco leucotis & 0 & 1 & 1 & 0 & 0 & 0 & 1 & 3 \\
\hline Tauraco livingstonii & 0 & 1 & 0 & 0 & 2 & 2 & 0 & 1 \\
\hline & & & & Ordem Pel & caniforme & & & \\
\hline Theristicus caudatus & 1 & 0 & 0 & 0 & 0 & 0 & 0 & 0 \\
\hline
\end{tabular}

Passeriformes 64,4\% (386/599), Psittaciformes 26,2\% (157/599), Musophagiformes 2\% (12/599), Tinamiformes 1,8\% (11/599), Piciformes 1,5\% (9/599), Phoenicopteriformes 1,0\% (6/599), Anseriformes e Gruiformes 0,8\% (5/599), Galliformes 0,6\% (4/599), Cuculiformes $0,3 \%$ $(2 / 599)$ e Pelecaniformes $0,2 \%(1 / 599)$.

Ao analisar o grupo das aves em geral, foram $28,05 \%$ resultados positivos (168/599) e 71,95\% resultados negativos (431/599), sendo 19,9\% (119/599) dos resultados obtidos no verão, 30,6\% (183/599) no outono, $28,04 \%$ (168/599) no inverno e $21,54 \%$ (129/599) na primavera. Dentro dos resultados obtidos, no verão 31,09\% (37/119) foram positivos; no outono 31,15\% (57/183); no inverno $30,4 \%(51 / 168)$ e por fim, na primavera $17,83 \%$ (23/129) 
(Quadro 3), sendo que nesta última estação a incidência foi significativamente menor ( $p=0,003$ ) (Quadro 4).

Como o número de indivíduos das ordens dos Passeriformes e Psittaciformes foi significativamente maior quando comparado ao de outras ordens, estas foram escolhidas para a análise estatística. A ordem dos Passeriformes, que foi a mais numerosa, tem como alguns representantes neste estudo o curió (Sporophila angolensis) e o canário-da-terra (Sicalis flaveola) (Peterson \& Life 1971, Marinho et al. 2010, Piacentini et al. 2015, Gill \& Donsker 2016). Foram 17,9\% (69/386) resultados positivos e $82,1 \%(317 / 386)$ resultados negativos, sendo 14,5\% (56/386) dos resultados pertencentes ao verão, $35,23 \%(136 / 386)$ ao outono, 31,9\% (123/386) ao inverno e 18,39\% (71/386) à primavera; dentro destes resultados, $14,3 \%(8 / 56)$ foram positivos no verão, $21,4 \%$ (29/136) no outono, $21,2 \%$ (26/123) no inverno e 8,5\% (6/71) na primavera (Quadro 5), também tendo sido demonstrada menor incidência nesta última estação $(p=0,02)$ (Quadro 6).

A ordem dos Psittaciformes, a segunda mais numerosa, possui apenas uma família, Psittacidae, tendo como alguns

Quadro 3. Distribuição do número de resultados positivos e negativos ( $\mathrm{N}$ ) encontrados entre 2010 e 2014 nas aves em geral de acordo com a estação do ano

\begin{tabular}{|c|c|c|c|c|c|c|c|c|c|c|}
\hline \multirow{2}{*}{$\begin{array}{l}\text { Aves } \\
\text { silvestres* }\end{array}$} & \multicolumn{2}{|c|}{ Verão } & \multicolumn{2}{|c|}{ Outono } & \multicolumn{2}{|c|}{ Inverno } & \multicolumn{2}{|c|}{ Primavera } & \multicolumn{2}{|c|}{ TOTAL } \\
\hline & $\mathrm{N}$ & $\%$ & $\mathrm{~N}$ & $\%$ & $\mathrm{~N}$ & $\%$ & $\mathrm{~N}$ & $\%$ & $\mathrm{~N}$ & $\%$ \\
\hline & 3 & & & & & & & & & \\
\hline & 82 & & 126 & & 117 & & 06 & & 1 &, 95 \\
\hline & 119 & 100 & 183 & 100 & 168 & 100 & 129 & 100 & 599 & 100 \\
\hline
\end{tabular}

*Aves pertencentes as ordens: Passeriforme, Psittaciforme, Musophagiforme, Tinamiforme, Piciforme, Phoenicopteriforme, Anseriforme, Gruiforme, Galliforme, Cuculiforme e Pelecaniforme.

Quadro 4. Distribuição dos resultados em partições para comparação, grau de liberdade (GL), valor encontrado de $x^{2}$ e de nível descritivo $(p)$ nas aves em geral

\begin{tabular}{ccccc}
\hline Partição & Lin:Col $^{\text {d }}$ & Qui-quadrado & GL f & (p) \\
\hline $1^{\text {a }}$ & $02: 02$ & 0,0001 & 1 & 0,9917 \\
$2^{\text {b }}$ & $02: 03$ & 0,0316 & 1 & 0,8589 \\
$3^{\text {c }}$ & $02: 04$ & 8,5046 & 1 & 0,0035 \\
Geral & $02: 05$ & 8,5364 & 3 & 0,0361
\end{tabular}

a Comparação entre verão e outono. Valor de p de 0,99 . Não houve diferença significativa entre as estações;

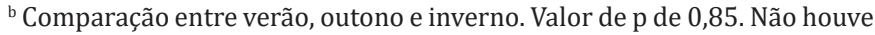
diferença significativa entre as estações;

' Comparação entre verão, outono, inverno e primavera. Valor de p de 0,03. Houve diferença altamente significativa ao comparar a primavera às demais estações, ou seja, na primavera a incidência de casos positivos foi significativamente menor;

${ }^{d}$ Comparação de linhas (positivos e negativos) com colunas ( $\mathrm{N}$ das estações).

Quadro 5. Distribuição do número de resultados positivos e negativos (N) encontrados entre 2010 e 2014 na ordem Passeriforme de acordo com a estação do ano

\begin{tabular}{|c|c|c|c|c|c|c|c|c|c|c|}
\hline \multirow{2}{*}{$\begin{array}{l}\text { Ordem } \\
\text { Passeriforme }\end{array}$} & \multicolumn{2}{|c|}{ Verão } & \multicolumn{2}{|c|}{ Outono } & \multicolumn{2}{|c|}{ Inverno } & \multicolumn{4}{|c|}{ Primavera TOTAL } \\
\hline & $\mathrm{N}$ & $\%$ & $\mathrm{~N}$ & $0 \%$ & $\mathrm{~N}$ & $\%$ & $N$ & $\%$ & $\mathrm{~N}$ & $\%$ \\
\hline & $\varepsilon$ & & & & & & & & & \\
\hline & 4 & & 7 & & 97 & 8 & 65 & 91,5 & 31 & 82,1 \\
\hline 101 & 56 & 100 & 136 & 100 & 123 & 100 & 71 & 100 & 386 & 100 \\
\hline
\end{tabular}

a Ordem Passeriforme, considerando as famílias Thraupidae e Fringilidae.
Quadro 6. Distribuição dos resultados em partições para comparação, grau de liberdade (GL), valor encontrado de $x^{2}$ e de nível descritivo $(p)$ na ordem dos Passeriformes

\begin{tabular}{lcccc}
\hline Partição & Lin:Col & Qui-quadrado & GL & $(p)$ \\
\hline $1^{\mathrm{a}}$ & $02: 02$ & 1,3383 & 1 & 0,2473 \\
$2^{\mathrm{b}}$ & $02: 03$ & 0,1781 & 1 & 0,673 \\
$3^{\mathrm{c}}$ & $02: 04$ & 5,2645 & 1 & 0,0218 \\
Geral & $02: 05$ & 6,7809 & 3 & 0,0792
\end{tabular}

${ }^{a}$ Comparação entre verão e outono. Valor de p de 0,24 . Não houve diferença significativa entre as estações;

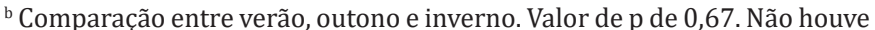
diferença significativa entre as estações;

c Comparação entre verão, outono, inverno e primavera. Valor de p de 0,02 . Houve diferença significativa ao comparar a primavera às demais estações, ou seja, na primavera a incidência de casos positivos foi significativamente menor

Quadro 7. Distribuição do número de resultados positivos e negativos (N) encontrados entre 2010 e 2014 na ordem Psittaciforme de acordo com a estação do ano

\begin{tabular}{|c|c|c|c|c|c|c|c|c|c|}
\hline Ordem & Verão & & tono & & verno & Pril & navera & & OTAL \\
\hline Psittaciforme & $\mathrm{N} \quad \%$ & $\mathrm{~N}$ & $\%$ & $\mathrm{~N}$ & $\%$ & $\mathrm{~N}$ & $\%$ & & $\%$ \\
\hline & & & 52 & & & & & & \\
\hline & 286 & 19 & 47,5 & 15 & 5 & 33 & 76 & 95 & 60,51 \\
\hline TOTAL & $45 \quad 100$ & 40 & 100 & 29 & 100 & 43 & 100 & 157 & 100 \\
\hline
\end{tabular}

Quadro 8. Distribuição dos resultados em partições para comparação, grau de liberdade (GL), valor encontrado de $x^{2}$ e de nível descritivo $(p)$ na ordem Psittaciforme

\begin{tabular}{ccccc}
\hline Partição & Lin:Col & Qui-quadrado & GL & $(p)$ \\
\hline $1^{\text {a }}$ & $02: 02$ & 1,9208 & 1 & 0,1658 \\
$2^{\text {b }}$ & $02: 03$ & 0,1153 & 1 & 0,7342 \\
$3^{\text {c }}$ & $02: 04$ & 6,5318 & 1 & 0,0106 \\
Geral & $02: 05$ & 8,5679 & 3 & 0,0356
\end{tabular}

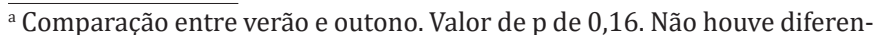
ça significativa entre as estações;

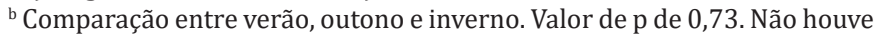
diferença significativa entre as estações;

' Comparação entre verão, outono, inverno e primavera. Valor de p de 0,01. Houve diferença muito significativa ao comparar a primavera às demais estações, ou seja, na primavera a incidência de casos positivos foi significativamente menor.

representantes neste estudo o papagaio-verdadeiro ( $A m a-$ zona aestiva), a arara-azul (Anodorhynchus hyacinthinus), a arara-canindé (Ara ararauna), a calopsita (Nymphicus hollandicus) e o papagaio do Senegal (Poicephalus senegalus) (Peterson \& Life 1971, Marinho et al. 2010, Piacentini et al. 2015, Gill \& Donsker 2016).

Foram $39,49 \%$ positivos $(62 / 157)$ e $60,51 \%$ resultados negativos (95/157), sendo estes resultados $28,66 \%$, (45/157) obtidos no verão, $25,48 \%$ (40/157) no outono, $18,47 \%$ (29/157) no inverno e $23,26 \%$ (43/157) resultados obtidos na primavera; dentro destes resultados, $37,78 \%(17 / 45)$ foram positivos no verão, $52,5 \%(21 / 40)$ positivos no outono, $48,28 \%(14 / 29)$ positivos no inverno e $23,26 \%(10 / 43)$ resultados positivos obtidos na primavera (Quadro 7). Neste grupo de aves também foi demonstrada menor incidência de resultados positivos para Candida spp. na primavera $(p=0,01)$ (Quadro 8).

A espécie dos curiós (Sporophila angolensis) (Peterson \& Life 1971, Marinho et al. 2010, Piacentini et al. 2015, Gill 
\& Donsker 2016) foi escolhida para análise por ser a mais numerosa dentre as outras deste estudo.

Nesta espécie, foram 14,94\% (46/308) resultados positivos e $85,06 \%(262 / 308)$ resultados negativos, sendo $13,96 \%$ (43/308) destes resultados obtidos no verão, $35,06 \%$ (108/308) obtidos no outono, 32,47\% (100/308) obtidos no inverno e 18,51\% (57/308) na primavera; dentro destes resultados, 9,3\% (4/43) foram positivos no verão, $16,67 \%$ (18/108) positivos no outono, 20\% (20/100) no inverno e 7,02\% (4/57) na primavera (Quadro 9). A in-

Quadro 9. Distribuição do número de resultados positivos e negativos (N) encontrados entre 2010 e 2014 na espécie Sporophila angolensis de acordo com a estação do ano

\begin{tabular}{|c|c|c|c|c|c|c|c|c|c|c|}
\hline Espécie & \multicolumn{2}{|c|}{ Verão } & \multicolumn{2}{|c|}{ Outono } & \multicolumn{2}{|c|}{ Inverno } & \multicolumn{2}{|c|}{ Primavera } & \multicolumn{2}{|c|}{ TOTAL } \\
\hline $\begin{array}{l}\text { Sporophila } \\
\text { angolensis }\end{array}$ & $\overline{\mathrm{N}}$ & $\%$ & $\overline{\mathrm{N}}$ & $\%$ & $\mathrm{~N}$ & $\%$ & $\mathrm{~N}$ & $\%$ & $\mathrm{~N}$ & $\%$ \\
\hline Positi & 4 & 9,3 & 18 & 16,67 & 20 & 20 & 4 & 7,02 & 46 & 14,94 \\
\hline Negativos & 39 & 90,7 & 90 & 83,33 & 80 & 80 & 53 & 92,98 & 262 & 85,06 \\
\hline TOTAL & 43 & 100 & 108 & 100 & 100 & 100 & 57 & 100 & 308 & 100 \\
\hline
\end{tabular}

Quadro 10. Distribuição dos resultados em partições para comparação, grau de liberdade (GL), valor encontrado de $\mathrm{x}^{2}$ e de nível descritivo $(p)$ na espécie Sporophila angolensis

\begin{tabular}{ccccc}
\hline Partição & Lin:Col & Qui-quadrado & GL & $(p)$ \\
\hline $1^{\text {a }}$ & $02: 02$ & 1,3129 & 1 & 0,2519 \\
$2^{\text {b }}$ & $02: 03$ & 1,3964 & 1 & 0,2373 \\
$3^{\text {c }}$ & $02: 04$ & 3,4512 & 1 & 0,0632 \\
Geral & $02: 05$ & 6,1605 & 3 & 0,1041
\end{tabular}

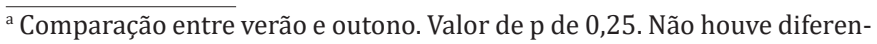
ça significativa entre as estações.

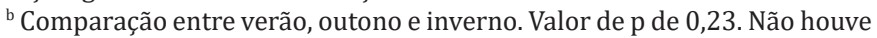
diferença significativa entre as estações;

Comparação entre verão, outono, inverno e primavera. Valor de p de 0,06. Não houve diferença significativa entre as estações.

Quadro 11. Distribuição do número de resultados positivos e negativos (N) encontrados entre 2010 e 2014 na região sudeste brasileira de acordo com a estação do ano

\begin{tabular}{|c|c|c|c|c|c|c|c|c|c|c|}
\hline \multirow{2}{*}{$\begin{array}{l}\text { Região } \\
\text { sudeste }\end{array}$} & \multicolumn{2}{|c|}{ Verão } & \multicolumn{2}{|c|}{ Outono } & \multicolumn{2}{|c|}{ Inverno } & \multicolumn{2}{|c|}{ Primavera } & \multicolumn{2}{|c|}{ TOTAL } \\
\hline & $\mathrm{N}$ & $\%$ & $\mathrm{~N}$ & $\%$ & $\mathrm{~N}$ & $\%$ & $\mathrm{~N}$ & $\%$ & $\mathrm{~N}$ & $\%$ \\
\hline & 3 & & 5 & & 39 & & & & 47 & \\
\hline & 67 & 0. & 4 & 6 & 92 & & 97 & ,2 & 70 & 71,57 \\
\hline ГОО & 103 & 100 & 165 & 100 & 131 & 100 & 118 & 100 & 517 & 100 \\
\hline
\end{tabular}

Quadro 12. Distribuição dos resultados em partições para comparação, grau de liberdade (GL), valor encontrado de $x^{2}$ e de nível descritivo $(p)$ na região sudeste brasileira

\begin{tabular}{ccccc}
\hline Partição & Lin:Col & Qui-quadrado & GL & $(p)$ \\
\hline $1^{\text {a }}$ & $02: 02$ & 0,5092 & 1 & 0,4755 \\
$2^{\text {b }}$ & $02: 03$ & 0,3133 & 1 & 0,5757 \\
$3^{\text {c }}$ & $02: 04$ & 8,501 & 1 & 0,0035 \\
Geral & $02: 05$ & 9,3236 & 3 & 0,0253
\end{tabular}

a Comparação entre verão e outono. Valor de p de 0,47. Não houve diferença significativa entre as estações;

${ }^{\text {b }}$ Comparação entre verão, outono e inverno. Valor de p de 0,57. Não houve diferença significativa entre as estações;

Comparação entre verão, outono, inverno e primavera. Valor de $\mathrm{p}$ de 0,003 . Houve diferença muito significativa ao comparar a primavera às demais estações, ou seja, na primavera a incidência de casos positivos foi significativamente menor. cidência de Candida spp. na primavera também foi menor, porém não significativa $(p=0,06)$ (Quadro 10).

Por fim, o último grupo analisado foi o de aves oriundas da região sudeste brasileira, uma vez que teve expressivos 517 resultados de 599 resultados totais (86,31\%), provavelmente devido ao fato do laboratório se localizar nesta região. Neste grupo foram 28,43\% (147/517) resultados positivos e 71,57\% (370/517) resultados negativos, sendo $19,92 \%(103 / 517)$ destes resultados obtidos no verão, $31,91 \%$ (165/517) no outono, $25,33 \%$ (131/517) no inverno e 22,82\% (118/517) obtidos na primavera; destes resultados, 34,95\% (36/103) foram positivos no verão, $30,9 \%$ (51/165) positivos no outono, 29,77\% (39/131) positivos no inverno e $17,8 \%$ (21/118) positivos na primavera (Quadro 11), também tendo sido demonstrada menor incidência na última estação $(p=0,003)$ (Quadro 12).

\section{DISCUSSÃO}

Em vista da epidemiologia do agente e dos resultados encontrados neste estudo, a incidência da doença parece estar intimamente relacionada com características do hospedeiro, uma vez que o micro-organismo é cosmopolita e altamente resistente a variações de temperatura e umidade, o que permitiria sua manifestação e adaptação às diversas áreas geográficas do país conforme evidenciado nos resultados, sendo habitante da microflora normal das mucosas principalmente, de aves e mamíferos e agindo como comensal (Hirsh \& Zee 2003, Fisher \& Cook 2001, Quinn et al. 2005, Silva 2010).

Nenhuma bibliografia pôde ser encontrada a respeito da menor incidência de Candida spp. na primavera nos grupos estudados, porém, sabe-se que a manifestação clínica da doença ocorre por desequilíbrio hormonal e imunológico, baixa resistência ao micro-organismo ou intensa exposição de hospedeiros debilitados ou tecidos vulneráveis, podendo ser disseminada por via hematógena na forma septicêmica para trato respiratório e intestino principalmente, além de outros órgãos eventualmente, sendo a resposta imune determinante para a forma de apresentação da doença causada por esta levedura (Hirsh \& Zee 2003, Fisher \& Cook 2001, Quinn et al. 2005, Silva 2010).

A sanidade das aves pode ser influenciada por fatores ambientais, comportamentais e nutricionais, como a temperatura, umidade, luminosidade e taxa de lotação do ambiente em que vivem e também devido a disponibilidade e qualidade dos alimentos e água ofertados no cativeiro, o que por consequência de um erro de manejo pode levar a um desequilíbrio hormonal e imunológico por estresse crônico e/ou também devido a uma eventual deficiência nutricional de macro minerais essenciais ao sistema imune quando é baixa a qualidade ou quantidade dos alimentos e água obtidos por estas aves, podendo levar a manifestação clínica de doenças causadas por fungos oportunistas (Schimidt et al. 2003, Carvalho et al. 2013, Fraga 2014).

No hemisfério sul, os meses de setembro, outubro e novembro são pertencentes à estação primavera, caracterizada pela aproximação ao solstício de verão, com aumento de temperatura nas latitudes médias, segundo o INMET (2015). De acordo com INPE (2015), em todas as regiões do Brasil, na primavera há uma intensificação das chuvas, que se tornam mais frequen- 
tes, sendo o período de transição, na maioria das regiões, da estação seca para a estação chuvosa. 0 aumento do calor e da umidade ocasionam pancadas de chuvas no final da tarde e/ou noite, aumentando gradativamente durante a estação.

Devido ao aumento do fotoperíodo, há maior disponibilidade de alimentos e, com clima quente tem-se início a reprodução das aves para melhor criação dos filhotes (Bays et al. 2009). Com estas mudanças, há um aumento no metabolismo destes animais, que começam a acumular mais energia para preparação do período reprodutivo e, na maioria das aves de vida livre, para migração, fenômeno muito desgastante, provocado por efeitos hipofisários em resposta a fatores climáticos. Há um desenvolvimento gonádico das aves para a produção de hormônios genitais e também para muda de plumagem pré-nupcial, com consequentes disputas entre machos pelas fêmeas; após estes episódios, há a cerimônia de galanteio. Para que as aves tenham sucesso nesta fase, é necessário que a plumagem esteja exuberante, o que envolve um grande gasto de energia para o preparo da muda. As aves neste período, com o aumento da intensidade de pluviosidade, também podem apreciar em abundância banhos diretamente das chuvas, no orvalho ou em acúmulos de água em folhagens. Além dos banhos de água, nesta época conseguem aproveitar melhor os banhos de sol, já que há aumento na incidência de raios solares (Peterson \& Life 1971, Ruschi 1979).

Portanto, levando-se em conta que há um aumento no metabolismo destas aves, devido as condições climáticas e consequentemente hormonais que a primavera traz, levando a um aumento no acúmulo de energia e a produção de hormônios gonadais para o preparo da época de reprodução, além do próprio fato de que nesta estação estas condições climáticas se tornam mais favoráveis para a criação de aves, que aumentam suas atividades sociais e consomem maior quantidade de alimentos com maior qualidade nutricional, espera-se que na primavera estas apresentem uma imunocompetência suficiente para proteção contra Candida spp., sendo necessários estudos práticos bioquímicos e hematológicos para confirmação efetiva desta hipótese.

\section{CONCLUSÃO}

Evidenciou-se neste estudo que, durante a primavera, na maioria dos grupos de aves analisados há diminuição da incidência de resultados positivos para Candida spp., possivelmente devido a um aumento da imunocompetência destes animais durante esta estação, sendo necessários mais estudos para associar resultados clínico-práticos aos estatísticos encontrados nesta pesquisa.

Agradecimentos.- Aos professores Lilian B. Elston e Marcelo L. Brisola, Pontifícia Universidade Católica de Minas Gerais, pela orientação e atenção dadas; ao Laboratório ADN, Poços de Caldas-Minas Gerais pela concessão dos laudos para a realização deste trabalho.

\section{REFERÊNCIAS}

Ayres M., Ayres J.R., Ayres D.L. \& Santos A.S. 2007. BioEstat 5.0 - aplicações estatísticas nas áreas das ciências biológicas e médicas: Sociedade Civil Mamirauá, Belém. CNPq, Brasília.

Bays T.B., Lightfoot T. \& Mayer J. 2009. Comportamento de Animais Exóticos de Companhia: Aves, Répteis e Mamíferos de Pequeno Porte. Roca, São Paulo.
Brandão J. \& Beaufrère H. 2013. Clinical update and treatment of selected infectious gastrointestinal diseases in avian species. J. Exot. Pet Med. 22:101-117.

Cafarchia C., Camarda A., Romito D., Campolo M., Quaglia N.C., Tullio D. \& Otranto D. 2006. Occurrence of yeasts in cloacae of migratory birds. Mycopathologia 161:229-234.

Carvalho C.D., Ramos J.D., Rameh-de-Albuquerque L.C., Silva M.A., Sousa E.L., Lustosa D.A. \& Soares P.C. 2013. Perfil hematológico, bioquímico sérico, proteína C reativa e cortisol de ararajubas (Guaroba guarouba) mantidas em cativeiro. Pesq. Vet. Bras. 33(3):394-398.

Coles B.H. 2007. Essentials of Avian Medicine and Surgery. 3rd ed. Blackwell Publishing, Oxford.

Fisher F. \& Cook N.B. 2001. Micologia: fundamentos e diagnóstico. Revinter, Rio de Janeiro.

Fraga C.F. 2014. Ocorrência de doenças micóticas em aves silvestres do Brasil. Trabalho de conclusão de Curso em Medicina Veterinária, Faculdade de Veterinária, Universidade Federal do Rio Grande do Sul, Porto Alegre, RS.

Gill F. \& Donsker D. 2016. IOC World Bird List (v 6.3). doi: 10.14344/IOC. ML.6.3

Hazen K.C. 1995. New and emerging yeast pathogens. Clin. Microbiol. Rev. 8(4):462-478.

Hirsh D.C. \& Zee Y.C. 2003. Microbiologia veterinária, p.103. In: Biberstein E.C. (Ed.), Candida. Vol.1. Guanabara Koogan, Rio de Janeiro.

INMET 2015. Data e Horário de Início das Estações do Ano. Instituto Nacional de Meteorologia, Brasília, DF.

INPE 2015. Estações do ano, Centro de Previsão de Tempo e Estudos Climáticos, São José dos Campos, SP.

Lanteri G., Macrì F., Rapisarda G., Basile F., Reale S. \& Marino F. 2012. Systemic candidiasis in farm-reared red-legged partridges (Alectoris rufa) caused by Leucosporidium spp. BMC Vet. Res. 8(81):2-4.

Machado A.B.M., Drummond G.M. \& Paglia A.P. 2010. Livro vermelho da fauna brasileira ameaçada de extinção, p.379. In: Silveira L.F. \& Straube F.C.(Eds), Aves Ameaçadas de Extinção no Brasil. Vol.2. Ministério do Meio Ambiente, Brasília.

Marinho M., Táparo C., Silva B., Tencate L. \& Perri S. 2010. Microbiota fúngica de passeriformes de cativeiros da região noroeste do estado de São Paulo. Vet. Zootec. 17(2):288-292.

Muir M. \& Raidal S.R. 2012. Necrotising ventriculitis due to combined infection with Rhizopus microsporus var. chinensis and Candida krusei in a ecletus parrot (Ecletus roratus). Aust. Vet. J. 90(7):277-280.

Nouri M., Movassaghi A.R. \& Kamyabi Z. 2009. Ventricular candidiasis in a lovebird (Agapornis fischeri). ESVP/ECVP Proceedings 141(4):301.

Piacentini V.Q., Aleixo A., Agne C.E., Maurício G.N., Pacheco J.F., Bravo G.A., Brito G.R., Naka L.N., Olmos F., Posso S., Silveira L.F., Betini G.S., Carrano E., Franz I., Lees A.C., Lima L.M., Pioli D., Schunck F., Amaral F.R., Bencke G.A., Cohn-Haft M., Figueiredo L.F., Straube F.C. \& Cesari E. 2015. Lista comentada das aves do Brasil pelo Comitê Brasileiro de Registros Ornitológicos. Revta Bras. Ornitol. 23(2):91-298.

Peterson R.T. \& Life N. 1971. As aves. Livraria José Olympio, Rio de Janeiro. Quinn P.J., Markey B.K., Leonard F.C. \& Carter M.E. 2005. Microbiologia Veterinária: e doenças infecciosas. Vol.1. Artmed, Porto Alegre.

Renctas 2001. 1ํ Relatório Nacional sobre o Tráfico de Fauna Silvestre. Vol.1. Renctas, Brasília.

Ruschi A. 1979. Aves do Brasil. Vol.1. Rios, São Paulo.

Santos L.L., Ferreira F.M., Lopes S.F., Condas L.A., Muro M.D. \& Lugarini C. 2009. Pesquisa de Cryptococcus neoformans e Candida spp. em excretas de psitacídeos e passeriformes cativos. Arq. Ciênc. Vet. Zool. Unipar 12(1):5-9.

Santos G.G.C., Matuella G.A., Coraiola A.M., Silva L.C.S., Lange R.R. \& Santin E. 2008. Doenças de aves selvagens diagnosticadas na Universidade Federal do Paraná (2003-2007). Pesq. Vet. Bras. 28(11):565-570.

Schimidt R.E., Reavill D.R. \& Phalen D.V. 2003. Pathology of Pet and Aviary Birds. Blackwell Publishing, Oxford.

Silva R.F. 2010. Infecções fúngicas em imunocrompometidos. J. Bras. Pneumol. 36(1):142-147.

Velasco M.C. 2000. Candidiasis and cryptococcosis in birds. Sem. Avian Exot. Pet Med. 9(2):75-81. 